\title{
Article \\ Integral Sliding Mode Anti-Disturbance Control for Markovian Jump Systems with Mismatched Disturbances
}

\author{
Hong Shen ${ }^{1, *}$, Xiaoli Zhang ${ }^{2}$ and Yang $\mathrm{Yi}^{2}$ \\ 1 Business College, Yangzhou University, Yangzhou 225009, China \\ 2 College of Information Engineering, Yangzhou University, Yangzhou 225009, China; \\ xiaolicontrol@163.com (X.Z.); yiyang@yzu.edu.cn (Y.Y.) \\ * Correspondence: shenhong@yzu.edu.cn
}

Citation: Shen, H.; Zhang, X.; Yi, Y. Integral Sliding Mode

Anti-Disturbance Control for

Markovian Jump Systems with

Mismatched Disturbances. Electronics

2021, 10, 1075. https:/doi.org/

10.3390/electronics10091075

Academic Editors: Hamid Reza Karimi, Cheng Siong Chin, Kalyana

C. Veluvolu, Valeri Mladenov, Cecilio Angulo, Davide Astolfi, Jun Yang and Len Gelman

Received: 29 March 2021

Accepted: 29 April 2021

Published: 2 May 2021

Publisher's Note: MDPI stays neutra with regard to jurisdictional claims in published maps and institutional affiliations.

Copyright: (c) 2021 by the authors. Licensee MDPI, Basel, Switzerland. This article is an open access article distributed under the terms and conditions of the Creative Commons Attribution (CC BY) license (https:// creativecommons.org/licenses/by/ $4.0 /)$.

\begin{abstract}
This paper addresses an integral sliding mode-based anti-disturbance control algorithm for a type of Markovian jump systems (MJSs), which are influenced by different types of mismatched disturbances. On one hand, as for those disturbances that can be modeled, the disturbance observer (DO) method is introduced to realize the dynamical estimation of disturbances. Based on this, both the integral sliding surface (ISS) and the composite anti-disturbance controller are proposed in succession for rejecting unknown disturbances and guaranteeing the stability of the controlled MJS. Meanwhile, the states of the controlled system are ensured to reach ISS within a finite time. In addition, the $L_{1}$ performance index is given to attenuate the effects of bounded disturbances. The controller and observer gains can be computed by using convex optimization techniques. The satisfactory stochastic stability and dynamical tracking performance are both also proved. Finally, the simulation results effectively verify all of the required performances.
\end{abstract}

Keywords: Markovian jump systems (MJSs); integral sliding mode (ISM) control; anti-disturbance control; mismatched disturbances

\section{Introduction}

In actual production practices, the state of the controlled system usually presents varying degrees of randomness, which will cause many difficulties in terms of system modeling and analysis [1]. Thus, the famous Markov jump systems (MJSs) are proposed. It is noted that MJSs are typical random systems with various modes, where the transition probability between modes is determined by Markov chain when the systems are running [2]. By using the dynamic characteristics of the Markov chains, MJSs can effectively simulate many types of engineering systems, such as manufacturing systems [3], solar systems [4], network-based control systems [5], and aerospace systems [6]. For example, a Markov random process needs to be imported to describe those random failures or disturbances of thrusters, sensors, and components in order to precise identify the dynamics of spacecraft. For a popular exoskeleton robot model, based on the angular position of four different Markovian states (body, shank, thigh, and feet), a Markovian estimation model is built to achieve the satisfactory dynamical description and information from each sensor [7]. Recently, the theoretical exploration on this kind of stochastic systems has also attracted considerable attentions of many scholars, and there have been a lot of results on the analysis of MJSs [8-14]. In [8], a sliding mode observer is designed to discuss the fault-tolerant problem for the MJSs with actuator fault. By using the T-S fuzzy model, Ref. [10] discusses a $L_{2}-L_{\infty}$ filtering design issue for a class of typical MJSs. In [12], the nonfragile $H_{\infty}$ control issue is considered for fast sampling discrete-time MJSs. Shen, Ma, Park, and Wang [13] concerns a fuzzy fault-tolerant control algorithm, which applies to MJSs with unknown mismatched faults.

As we all know, exogenous disturbances can be found in nearly all engineering applications, which severely affects the property of the controlled systems [15]. With the de- 
velopment of control science, the anti-disturbance issue has undoubtedly become a research hot issue. Many effective anti-disturbance methods have been widely used, such us active disturbance rejection control (ADRC) [16,17], robust control [18], and disturbance observer based control (DOBC) $[19,20]$, etc. Among them, the DOBC method aims at completely canceling disturbance to ensure the stability of the controlled system when disturbance occurs. In addition, DOBC has also been widely applied into actual engineering systems, such as robot models [21], spacecraft systems [22,23] and permanent magnet synchronous motors [24], DC-DC buck converters [25], and chemical distribution systems [26], due to the simplicity of the implementation and the flexible control structure. Hence, no matter the perspective of theory or application, DOBC has a certain research value. Yi, Zheng, and Liu [27] proposes an adaptive anti-disturbance control balgorithmy using dynamical neural network disturbance modelling. In [28], a generalized disturbance observer (DO) is considered to be able to estimate higher-order disturbances in the expanded time series. Yao, Park, Wu, and Guo [29] proposes a DOBC anti-disturbance method for the singular MJSs. In [30,31], the extended high-gain observer is designed to estimate the perturbation and further apply it to the multi-agent systems.

In existing control theories, SMC has always been a vaild nonlinear robust control technique and received considerable attention [32-35]. The design of sliding mode has nothing to do with system parameters and external disturbances, which is to say, it can be designed artificially. Accordingly, the SMC method has strong robustness. Huynh, Minh, Amaefule, Tran, and Tran [36] focuses on the design of high order sliding mode observer based power and load frequency control of multi-area interconnected power systems. Based on the existing SMC, a nonsingular terminal SMC is discussed in [37], which can get to the designed sliding surface in finite time without nonsingular phenomenon. However, in general SMC, it will be very sensitive against disturbances or uncertainties once reaching the sliding surface. Therefore, an ISM control scheme is designed to drive the initial state into the sliding surface and eliminate the arrival phase [38]. Wang, Shen, Karim, and Duan [39] discusses a fuzzy ISM control scheme for the typical T-S fuzzy models with matched disturbances. In [40], an ISM control method is investigated for the robot systems with time-delay estimation. As for the uncertain singularly perturbed system affected by disturbances, the passive ISM control and $\varepsilon$-bound estimation problem are discussed in [41].

According to the previous analysis, this manuscript pays attention to the research of ISM-based anti-disturbance control for the MJSs suffering with mismatched disturbances. It is noticed that most of results of MJSs cannot directly compensate the effects of mismatched disturbances. Few discussions pay attention to the dynamical tracking and output constraint problem (see [9-12,29] for details). When compared with previous results, two different mismatched disturbances are considered in the controlled MJSs. For the disturbances that can be modeled or part of the information can be measured, a DO is built to effectively estimate the dynamics of disturbances. According to observation information and feedback state, an ISS and the corresponding ISM control input are designed to realize the real-time compensation of unmatched disturbances. For those unmodelable disturbances, $L_{1}$ performance index is imported to restrain the influence of disturbance on measurement output. By using convex optimization method, the augmented MJSs can be proved to be stochastic stable. Meanwhile, the tracking error can be proved to converge to zero and the output constraint is also verified. Finally, the simulation examples are presented to substantiate the effectiveness of the proposed algorithm.

Notation: $I$ and 0 , respectively, represent a identity matrix and a zero matrix; For a vector $\gamma,\|\cdot\|$ represents $\|\gamma\|=\sqrt{\gamma^{T} \gamma} ; \lambda_{\min }$ stands for the minimum eigenvalue; For a matrix $Q, \operatorname{sym}\{Q\}$ can be described as $\operatorname{sym}\{Q\}=Q^{T}+Q$. 


\section{System Description}

In the section, a class of MJSs with mismatched disturbances is considered as

$$
\left\{\begin{aligned}
\dot{x}(t) & =A\left(r_{t}\right) x(t)+B\left(r_{t}\right) u(t)+C\left(r_{t}\right) h(t)+C_{d}\left(r_{t}\right) d(t) \\
y(t) & =D\left(r_{t}\right) x(t) \\
y_{d}(t) & =D_{d}\left(r_{t}\right) x(t)+E_{d}\left(r_{t}\right) d(t)
\end{aligned}\right.
$$

where $x(t) \in R^{m}, y(t) \in R^{n_{1}}, y_{d}(t) \in R^{n_{2}}$, and $u(t) \in R^{p}$ are the system state, the system output, the measurable output, and the controlled input, respectively. $r_{t}$ stands for continuous Markovian process, which takes a value on a finite state set $\aleph=\{1, \cdots, N\}$. Afterwards, the transfer rate matrix $\Pi=\left[\pi_{i j}\right]_{i, j \in \aleph}$ is expressed as

$$
\operatorname{Pr}\left\{r_{t+\triangle}=j \mid r_{t}=i\right\}= \begin{cases}1+\pi_{i j} \triangle+o(\triangle), & i=j \\ \pi_{i j} \triangle+o(\triangle), & i \neq j\end{cases}
$$

where $\lim _{\triangle \rightarrow 0} \frac{o(\Delta)}{\triangle}=0, \triangle>0 . \pi_{i j}$ is the transition ratio jumping from mode $i$ to mode $j$. For any $i \in \aleph, \pi_{i i}=-\sum_{j=1, i \neq j}^{N} \pi_{i j}$ is satisfied. $A\left(r_{t}\right), B\left(r_{t}\right), C\left(r_{t}\right), D\left(r_{t}\right), D_{d}\left(r_{t}\right)$, and $E_{d}\left(r_{t}\right)$ are the system matrices with suitable dimension. Subsequently, define $A\left(r_{t}\right):=A_{i}$, $B\left(r_{t}\right):=B_{i}, C\left(r_{t}\right):=C_{i}, C_{d}\left(r_{t}\right):=C_{d i}, D\left(r_{t}\right):=D_{i}, D_{d}\left(r_{t}\right):=D_{d i}$, and $E_{d}\left(r_{t}\right):=E_{d i}$.

Please note that $d(t) \in R^{p}$ and $h(t) \in R^{p}$ are two kinds of different disturbances, where $d(t)$ is supposed to be bounded with its norm. Choosing different upper bounds has no substantial impact on the analysis of the system. In order to simplify the proof process, $\|d(t)\|_{\infty} \leq 1$ holds. While another external disturbance $h(t)$ is assumed to be generated by the epitaxial system, as

$$
\left\{\begin{array}{l}
h(t)=V_{i} \sigma(t) \\
\dot{\sigma}(t)=W_{i} \sigma(t)
\end{array}\right.
$$

where $\sigma(t) \in R^{q}$ is the state of the disturbance model. $V_{i}$ and $W_{i}$ are the known matrices.

For achieving favoable dynamic performance, the state is extended as

$$
\bar{x}(t)=\left[x^{T}(t), \int_{0}^{t} e^{T}(\vartheta) d \vartheta\right]^{T}
$$

where the error term $e(t)$ is defined as $e(t):=y(t)-y_{h}$, and $y_{h}$ is the desired output.

By integrating (1) with (4), the expanded MJS is further described as

$$
\left\{\begin{aligned}
\dot{\bar{x}}(t) & =\bar{A}_{i} \bar{x}(t)+\bar{B}_{i} u(t)+\bar{C}_{i} h(t)+\bar{C}_{d i} d(t)+\bar{H}_{i} y_{h} \\
y(t) & =\bar{D}_{i} \bar{x}(t) \\
y_{d}(t) & =\bar{D}_{d i} \bar{x}(t)+\bar{E}_{d i} d(t)
\end{aligned}\right.
$$

where

$$
\begin{aligned}
& \bar{A}_{i}=\left[\begin{array}{cc}
A_{i} & 0 \\
C_{i} & 0
\end{array}\right], \quad \bar{B}_{i}=\left[\begin{array}{c}
B_{i} \\
0
\end{array}\right], \quad \bar{C}_{d i}=\left[\begin{array}{c}
C_{d i} \\
0
\end{array}\right], \quad \bar{C}_{i}=\left[\begin{array}{c}
C_{i} \\
0
\end{array}\right] \\
& \bar{D}_{i}=\left[\begin{array}{c}
D_{i}^{T} \\
0
\end{array}\right]^{T}, \quad \bar{D}_{d i}=\left[\begin{array}{c}
D_{d i}^{T} \\
0
\end{array}\right], \quad \bar{H}_{i}=\left[\begin{array}{c}
0 \\
-I
\end{array}\right], \bar{E}_{d i}=E_{d i}
\end{aligned}
$$

The following conditions need to be met in order to achieve feasible results.

Assumption 1. $\left(A_{i}, B_{i}\right)$ is controllable, and $\left(W_{i}, B_{i}, V_{i}\right)$ is observable.

Assumption 2. The disturbance $h(t)$ is assumed to be bounded and satisfies the condition $\|h(t)\| \leq \rho$, where $\rho>0$ is a defined scalar. 


\section{Design of Disturbance Observer}

For the sake of reconstructing disturbance $h(t)$ in MJSs, an effective DO is defined as

$$
\left\{\begin{array}{l}
\hat{h}(t)=V_{i} \hat{\sigma}(t) \\
\hat{\sigma}(t)=\omega(t)-L_{i} \bar{x}(t) \\
\left.\dot{\omega}(t)=L_{i}\left(\bar{A}_{i} \bar{x}(t)+\bar{B}_{i} u(t)+\bar{H}_{i} y_{h}\right)+\left(W_{i}+L_{i} \bar{C}_{i} V_{i}\right)\left(\omega(t)-L_{i} \bar{x}(t)\right)\right)
\end{array}\right.
$$

where $\omega(t)$ is a given auxiliary variable. $\hat{h}(t)$ and $\hat{\sigma}(t)$, respectively, stand for the estimations of $h(t)$ and $\sigma(t) . L_{i}$ is a gain matrix of DO, which will be solved later.

Define $e_{\sigma}(t)=\sigma(t)-\hat{\sigma}(t)$, then we have

$$
\dot{e}_{\sigma}(t)=\left(W_{i}+L_{i} \bar{C}_{i} V_{i}\right) e_{\sigma}(t)+L_{i} \bar{C}_{d i} d(t)
$$

The next theorem 1 concerns the dynamical performance of the estimated error system (7).

Theorem 1. For a given parameter $\lambda_{1}>0$, if there exists matrices $P_{1 i}>0$ and $T_{1 i}, i=1,2, \cdots, N$, which can ensure the inequalities

$$
\left[\begin{array}{cc}
\operatorname{sym}\left\{P_{1 i} W_{i}+T_{1 i} C_{i} V_{i}\right\}+\sum_{j=1}^{N} \pi_{i j} P_{1 j} & T_{1 i} \bar{C}_{d i} \\
* & -\lambda_{1}^{2} I
\end{array}\right]<0, \quad i=1, \cdots, N
$$

hold, then it can be proved that the estimation error system (7) is stochastically stable. The DO gain $L_{i}$ can be calculated by $L_{i}=P_{1 i}^{-1} T_{1 i}$.

Proof of Theorem 1. Select the Lyapunov function as

$$
\Phi_{1}\left(e_{\sigma}(t), i, t\right)=e_{\sigma}^{T}(t) P_{1 i} e_{\sigma}(t)
$$

Next, define the symbol $\digamma$ as the asthenic infinitesimal generator. By the total probability and conditional expectation, it is deduced that

$$
\begin{aligned}
\digamma \Phi_{1}\left(e_{\sigma}(t), i, t\right)= & \lim _{\triangle \rightarrow 0} \frac{1}{\triangle}\left\{\sum_{j=1, j \neq i}^{N} \operatorname{Pr}\left\{r_{t+\delta}=j \mid r_{t}=i\right\} e_{\sigma}^{T}(t+\triangle) P_{1 j} e_{\sigma}(t+\triangle)\right. \\
& \left.+\operatorname{Pr}\left\{r_{t+h}=i \mid r_{t}=i\right\} e_{\sigma}^{T}(t+\triangle) P_{1 i} e_{\sigma}(t+\triangle)-e_{\sigma}^{T}(t) P_{1 i} e_{\sigma}(t)\right\} \\
= & \lim _{\triangle \rightarrow 0} \frac{1}{\triangle}\left\{\sum_{j=1, j \neq i}^{N} \frac{\kappa_{i j}\left(G_{i}(\delta+\triangle)-G_{i}(\delta)\right)}{1-G_{i}(h)} e_{\sigma}^{T}(t+\triangle) P_{1 j} e_{\sigma}(t+\triangle)\right. \\
& \left.+\frac{1-G_{i}(\delta+\triangle)}{1-G_{i}(\delta)} e_{\sigma}^{T}(t+\triangle) P_{1 i} e_{\sigma}(t+\triangle)-e_{\sigma}^{T}(t) P_{1 i} e_{\sigma}(t)\right\}
\end{aligned}
$$

where $\delta$ is the time that is maintained on mode $i$ before moving to mode $j$. Besides, $\kappa_{i j}$ denotes the probability from mode $i$ to mode $j . G_{i}(\sigma)$ stands for the cumulative distribution function of $\sigma$ on mode $i$.

For a slightly positive number $\triangle$, according to Taylor formula, one has

$$
\begin{aligned}
e_{\sigma}(t+\triangle) & =e_{\sigma}(t)+\dot{e}_{\sigma}(t) \triangle+o(\triangle) \\
& =\left\{\left(W_{i}+L_{i} \bar{C}_{i} V_{i}\right) \triangle+I\right\} e_{\sigma}(t)+L_{i} \bar{C}_{d i} d(t) \triangle+o(\triangle)
\end{aligned}
$$


Further, based on (10) and (11), it is derived that

$$
\begin{aligned}
\digamma \Phi_{1}\left(e_{\sigma}(t), i, t\right) & =e_{\sigma}^{T}(t) \Pi e_{\sigma}(t)+2 e_{\sigma}^{T}(t) P_{1 i} L_{i} \bar{C}_{d i} d(t) \\
& \leq e_{\sigma}^{T}(t)\left[\Pi+\lambda_{1}^{-2} P_{1 i} L_{i} \bar{C}_{d i} \bar{C}_{d i}^{T} L_{i}^{T} P_{1 i}\right] e_{\sigma}(t)+\lambda_{1}^{2}
\end{aligned}
$$

where

$$
\begin{aligned}
\Pi=\operatorname{sym}\left\{P_{1 i} W_{i}+\right. & \left.P_{1 i} L_{i} C_{i} V_{i}\right\}+\lim _{\triangle \rightarrow 0} \sum_{j=1, j \neq i}^{N} \frac{\kappa_{i j}\left(G_{i}(\delta+\triangle)-G_{i}(\delta)\right)}{\triangle\left(1-G_{i}(\delta)\right)} P_{1 j} \\
& +\lim _{\triangle \rightarrow 0} \frac{G_{i}(\delta)-G_{i}(\delta+\triangle)}{\triangle\left(1-G_{i}(\delta)\right)} P_{1 i}
\end{aligned}
$$

By using the techniques of cumulative distribution functions, $\lim _{\triangle \rightarrow 0} \frac{G_{i}(\delta)-G_{i}(\delta+\Delta)}{\Delta\left(1-G_{i}(\delta)\right)}=\pi_{i}$ can hold, where $\pi_{i}$ denotes the transition rate when jumping from mode $i$.

Define $\pi_{i j}=\kappa_{i j} \pi_{i}, j \neq i$ and $\pi_{i i}=-\sum_{j=1, i \neq j}^{N} \pi_{i j}$. Afterwards, we obtain

$$
\digamma \Phi_{1} \leq e_{\sigma}^{T}(t)\left(\operatorname{sym}\left\{P_{1 i} W_{i}+P_{1 i} L_{i} C_{i} V_{i}\right\}+\sum_{j=1}^{N} \pi_{i j} P_{1 j}+\lambda_{1}^{-2} P_{1 i} L_{i} \bar{C}_{d i} \bar{C}_{d i}^{T} L_{i}^{T} P_{1 i}\right) e_{\sigma}(t)+\lambda_{1}^{2}
$$

According to (8), there exits a positive number $\chi_{1}$ satifying

$$
\digamma \Phi_{1}\left(e_{\sigma}(t), i, t\right) \leq-\chi_{1} e_{\sigma}^{T}(t) e_{\sigma}(t)+\lambda_{1}^{2}
$$

It can be gotten that, if $\left\|e_{\sigma}(t)\right\|^{2}>\chi_{1}^{-1} \lambda_{1}^{2}$ holds, $\digamma \Phi_{1}\left(e_{\sigma}(t), i, t\right)<0$ is guaranteed. As a result, the disturbance estimation error can be verified to be unanimously ultimately bounded and the augmented closed-loop system (7) is also stochastically stable.

\section{Design of ISS and ISM Controller}

By using the observation information of $h(t)$, design the ISS, as follows

$$
s(t)=\bar{F}_{i} \bar{x}(t)-\bar{F}_{i} \int_{0}^{t}\left(\bar{A}_{i} \bar{x}(\tau)+\bar{B}_{i} u_{1}(\tau)+\bar{C}_{i} \hat{h}(t)+\bar{H}_{i} y_{h}\right) d \tau
$$

where $\bar{F}_{i}$ are the designed matrices with appropriate dimension. $\bar{F}_{i} \bar{B}_{i}$ are assumed to be non-singular.

Based on (15), the nominal control input $u_{1}(t)$ is conducted as

$$
u_{1}(t)=K_{i} \bar{x}(t)-K_{h i} \hat{h}(t)
$$

where $K_{i}$ is the gains of controller. $K_{h i}$ is the disturbance suppression gains, which is expressed as $K_{h i}=\bar{B}_{i}^{+} \bar{C}_{i}, \bar{B}_{i}^{+}=\left(\bar{B}_{i}^{T} \bar{B}_{i}\right)^{-1} \bar{B}_{i}^{T}$.

The discontinuous control law $u_{2}(t)$ will be considered in order to make sure the trajectories of augmented system (5) enter into the sliding surface. Subsequently, the ISM control inout is designed as

$$
u(t)=u_{1}(t)+u_{2}(t)
$$

For the sake of finding the suitable $u_{2}(t)$, the following assumption needs to be met.

Assumption 3. The estimation error term $e_{h}(t)=h(t)-\hat{h}(t)$ is supposed to be bounded, and satisfies $\left\|e_{h}(t)\right\| \leq \varepsilon$, where $\varepsilon>0$ is a designed constant. 
Subsequently, $u_{2}(t)$ is designed as

$$
u_{2}(t)=-\alpha \frac{\bar{B}_{i}^{T} \bar{F}_{i}^{T} s(t)}{\left\|\bar{B}_{i}^{T} \bar{F}_{i}^{T}\right\|\|s(t)\|}-\left(\bar{F}_{i} \bar{B}_{i}\right)^{-1} \frac{\bar{F}_{i} \bar{C}_{i} \bar{C}_{i}^{T} \bar{F}_{i}^{T} s(t)}{\left\|\bar{C}_{i}^{T} \bar{F}_{i}^{T}\right\|\|s(t)\|} \varepsilon-\left(\bar{F}_{i} \bar{B}_{i}\right)^{-1} \frac{\bar{F}_{i} \bar{C}_{d i} \bar{C}_{d i}^{T} \bar{F}_{i}^{T} s(t)}{\left\|\bar{C}_{d i}^{T} \bar{F}_{i}^{T}\right\|\|s(t)\|}
$$

where $\alpha$ is a positive scalar.

In the following, the derivation of (15) is deduced as

$$
\dot{s}(t)=\bar{F}_{i}\left(\bar{B}_{i}\left(u(t)-u_{1}(t)\right)+\bar{C}_{i} e_{h}(t)+\bar{C}_{d i} d(t)\right)
$$

Substitute the designed ISM controller (16) into (19), one has

$$
\dot{s}(t)=\bar{F}_{i}\left(\bar{B}_{i} u_{2}(t)+\bar{C}_{i} e_{h}(t)+\bar{C}_{d i} d(t)\right)
$$

The following theorem will discuss the reachability problem of the state of the expanded system (5).

Theorem 2. When considering the designed ISM controller (16), the state trajectories of the controlled MJSs (5) are proved to reach the designed ISS (15) in a finite time $T_{t}$, where

$$
T_{t} \leq \frac{\sqrt{2 \bar{F}_{i} \bar{x}(0)}}{\alpha\left\|\bar{B}_{i}^{T} \bar{F}_{i}^{T}\right\|}
$$

Proof of Theorem 2. Select the Lyapunov function as

$$
\Phi_{2}(s, i, t)=\frac{1}{2} s^{T}(t) s(t)
$$

It is easy to compute the first order approximation of $s(t+\triangle)$, which can be expressed as

$$
s(t+\triangle)=s(t)+\dot{s}(t) \triangle+o(\triangle)
$$

Subsequently, based on the total probability, conditional expectation, and (22), the asthenic infinitesimal generator of $\Phi_{2}$ is deduced as

$$
\begin{aligned}
\digamma \Phi_{2}(s, i, t)= & \lim _{\triangle \rightarrow 0} \frac{1}{\triangle}\left\{\sum_{j=1, i \neq j}^{N} \operatorname{Pr}\left\{r_{t+\delta}=j \mid r_{t}=i\right\} s^{T}(t+\triangle) s(t+\triangle)\right. \\
& \left.+\operatorname{Pr}\left\{r_{t+h}=i \mid r_{t}=i\right\} s^{T}(t+\triangle) s(t+\triangle)-s^{T}(t) s(t)\right\} \\
= & \lim _{\triangle \rightarrow 0} \frac{1}{\triangle}\left\{\sum_{j=1, j \neq i}^{N} \frac{\kappa_{i j}\left(G_{i}(\delta+\triangle)-G_{i}(\delta)\right)}{1-G_{i}(h)} s^{T}(t+\triangle) s(t+\triangle)\right. \\
& \left.+\frac{1-G_{i}(\delta+\triangle)}{1-G_{i}(\delta)} s^{T}(t+\triangle) s(t+\triangle)-s^{T}(t) s(t)\right\} \\
= & 2 s^{T}(t) \dot{s}(t)+\sum_{j=1}^{N} \pi_{i j} \\
= & 2 s^{T}(t) \dot{s}(t)
\end{aligned}
$$


According to (18) and (20), one has

$$
\begin{aligned}
\digamma \Phi_{2}(s(t), i, t)= & s^{T}(t)\left(\bar{F}_{i} \bar{B}_{i} u_{2}(t)+\bar{F}_{i} \bar{C}_{i} e_{h}(t)+\bar{F}_{i} \bar{C}_{d i} d(t)\right) \\
= & -\alpha\left\|\bar{B}_{i}^{T} \bar{F}_{i}^{T}\right\|\|s(t)\|-\left\|\bar{C}_{i}^{T} \bar{F}_{i}^{T}\right\|\|s(t)\| \varepsilon+s^{T}(t) \bar{F}_{i} \bar{C}_{i} e_{h}(t) \\
& -\left\|\bar{C}_{d i}^{T} \bar{F}_{i}^{T}\right\|\|s(t)\|+s^{T}(t) \bar{F}_{i} \bar{C}_{d i} d(t) \\
\leq & -\alpha\left\|\bar{B}_{i}^{T} \bar{F}_{i}^{T}\right\|\|s(t)\|=-\alpha\left\|\bar{B}_{i}^{T} \bar{F}_{i}^{T}\right\| \sqrt{2 \Phi_{2}}
\end{aligned}
$$

It can be deduced that for any $t \geq T_{t}$, there exits

$$
T_{t} \leq \frac{2 \sqrt{\Phi_{2}(0)}}{\alpha\left\|\bar{B}_{i}^{T} \bar{F}_{i}^{T}\right\|}=\frac{2 \sqrt{s(0)}}{\alpha\left\|\bar{B}_{i}^{T} \bar{F}_{i}^{T}\right\|}=\frac{\sqrt{2 \bar{F}_{i} \bar{x}(0)}}{\alpha\left\|\bar{B}_{i}^{T} \bar{F}_{i}^{T}\right\|}
$$

To sum up, it is easy to see that the state of the expanded MJSs (5) can arrive the ISS in a finite time.

Solve $\dot{s}(t)=0$, then we can obtain the equivalent form of $u_{2}(t)$, as follows

$$
u_{2}^{e q}(t)=-\bar{B}_{i}^{+} \bar{C}_{i} e_{h}(t)-\bar{B}_{i}^{+} \bar{C}_{d i} d(t)
$$

and

$$
u^{e q}(t)=u_{1}(t)+u_{2}^{e q}(t)
$$

Remark 1. The structure of controller (17) includes two parts. Please note that u1(t) is a common feedback control, which can compensate the disturbance and guarantee the controlled system stability. While $u_{2}(t)$ is the discontinuous control that makes the state reach the designed ISS. In the Theorem 2, the theoretical proof is given to embody the purpose of $u_{2}(t)$. To my knowledge, the similar controller design method can be found in most of classical sliding mode control results and it is also proved to be effective.

By integrating (5) with (28), the dynamics of the system states on the ISS are described by

$$
\begin{aligned}
\dot{\bar{x}}(t) & =\bar{A}_{i} x(t)+\bar{B}_{i} u^{e q}(t)+\bar{C}_{i} d(t)+\bar{H}_{i} y_{h} \\
& =\left(\bar{A}_{i}+\bar{B}_{i} K_{i}\right) \bar{x}(t)+\left(\bar{C}_{i}-\bar{B}_{i} \bar{B}_{i}^{+} \bar{C}_{i}\right) h(t)+\left(\bar{C}_{d i}-\bar{B}_{i} \bar{B}_{i}^{+} \bar{C}_{d i}\right) d(t)+\bar{H}_{i} y_{h}
\end{aligned}
$$

\section{Dynamical Performance Analysis}

Theorem 3. For given parameters $\lambda_{1}>0, \lambda_{2}>0$, and $\lambda_{3}>0$, if we can find matrices $Q_{i}=P_{2 i}^{-1}$ and $T_{2 i}, i=1,2, \cdots, N$ to make the following inequalities

$$
\left[\begin{array}{cccc}
\operatorname{sym}\left\{\bar{A}_{i} Q_{i}+\bar{B}_{i} T_{2 i}\right\}+Q_{i} & \bar{C}_{i}-\bar{B}_{i} \bar{B}_{i}^{+} \bar{C}_{i} & \bar{C}_{d i}-\bar{B}_{i} \bar{B}_{i}^{+} \bar{C}_{d i} & \bar{H}_{i} \\
* & -\lambda_{1}^{-2} I & 0 & 0 \\
* & * & -\lambda_{2}^{-2} I & 0 \\
* & * & * & -\lambda_{3}^{-2} I
\end{array}\right]<0, i=1, \cdots, N
$$

are solvable, then the closed-loop MJSs (5) can be proved to be stable and the state trajectory will fall into a designed bicompact set $\Omega_{\bar{x}(t)}$, where $\Omega_{\bar{x}}=\left\{\bar{x} \mid\|\bar{x}(t)\| \leq \lambda_{\text {min }}^{-1}\left(P_{2 i}\right) \zeta\right\}$, $\zeta=\max \left\{\bar{x}^{T}(0) P_{2 i} \bar{x}(0), \lambda_{1}^{2} \rho^{2}+\lambda_{2}^{2}+\lambda_{3}^{2} y_{h}^{2}\right\}$.

Proof of Theorem 3. Select a suitable Lyapunov condition as

$$
\Phi_{3}(\bar{x}, i, t)=\bar{x}^{T}(t) P_{2 i} \bar{x}(t)
$$

Similar to (11) and (23), the first-order approximation of $\bar{x}(t+\triangle)$ is computed as

$$
\bar{x}(t+\triangle)=\dot{\bar{x}}(t) \triangle+\bar{x}(t)+o(\triangle)
$$


Subsequently, the asthenic infinitesimal generator of $\Phi_{3}$ can be obtained as

$$
\begin{aligned}
\digamma \Phi_{3}(\bar{x}, i, t)= & \lim _{\triangle \rightarrow 0} \frac{1}{\triangle}\left\{\sum_{j=1, j \neq i}^{N} \operatorname{Pr}\left\{r_{t+\delta}=j \mid r_{t}=i\right\} \bar{x}^{T}(t+\triangle) P_{2 j} \bar{x}(t+\triangle)\right. \\
& \left.+\operatorname{Pr}\left\{r_{t+h}=i \mid r_{t}=i\right\} \bar{x}^{T}(t+\triangle) P_{2 i} \bar{x}(t+\triangle)-\bar{x}^{T}(t) P_{2 i} \bar{x}(t)\right\} \\
= & \lim _{\triangle \rightarrow 0} \frac{1}{\triangle}\left\{\sum_{j=1, j \neq i}^{N} \frac{\kappa_{i j}\left(G_{i}(\delta+\triangle)-G_{i}(\delta)\right)}{1-G_{i}(h)} \bar{x}^{T}(t+\triangle) P_{2 j} \bar{x}(t+\triangle)\right. \\
& \left.+\frac{1-G_{i}(\delta+\triangle)}{1-G_{i}(\delta)} \bar{x}^{T}(t+\triangle) P_{2 i} \bar{x}(t+\triangle)-\bar{x}^{T}(t) P_{2 i} \bar{x}(t)\right\} \\
= & 2 \bar{x}^{T}(t) P_{2 i} \dot{\bar{x}}(t)+\sum_{j=1}^{N} \pi_{i j} P_{2 j}
\end{aligned}
$$

Substituting (29) into (33), it can be deduced as

$$
\begin{aligned}
\digamma \Phi_{3}(\bar{x}, i, t)= & 2 \bar{x}^{T} P_{2 i}\left(\bar{A}_{i}+\bar{B}_{i} K_{i}\right) \bar{x}+2 \bar{x}^{T}(t) P_{2 i}\left(\bar{C}_{i}-\bar{B}_{i} \bar{B}_{i}^{+} \bar{C}_{i}\right) h(t) \\
& +2 \bar{x}^{T} P_{2 i}\left(\bar{C}_{d i}-\bar{B}_{i} \bar{B}_{i}^{+} \bar{C}_{d i}\right) d(t)+2 \bar{x}^{T}(t) P_{2 i} \bar{H}_{i} y_{h}+\sum_{j=1}^{N} \pi_{i j} P_{2 j} \\
\leq & \bar{x}^{T}(t) \Gamma \bar{x}(t)+\lambda_{1}^{2}\|d(t)\|^{2}+\lambda_{2}^{2}\|h(t)\|^{2}+\lambda_{3}^{2} y_{h}^{2} \\
\leq & \bar{x}^{T}(t) \Gamma \bar{x}(t)+\lambda_{1}^{2} \rho^{2}+\lambda_{2}^{2}+\lambda_{3}^{2} y_{h}^{2}
\end{aligned}
$$

where

$$
\left\{\begin{aligned}
O_{i} & =\left(\bar{C}_{i}-\bar{B}_{i} \bar{B}_{i}^{+} \bar{C}_{i}\right)\left(\bar{C}_{i}-\bar{B}_{i} \bar{B}_{i}^{+} \bar{C}_{i}\right)^{T} \\
O_{d i} & =\left(\bar{C}_{d i}-\bar{B}_{i} \bar{B}_{i}^{+} \bar{C}_{d i}\right)\left(\bar{C}_{d i}-\bar{B}_{i} \bar{B}_{i}^{+} \bar{C}_{d i}\right)^{T} \\
\Gamma & =\operatorname{sym}\left\{P_{2 i}\left(\bar{A}_{i}+\bar{B}_{i} K_{i}\right)\right\}+\lambda_{1}^{-2} P_{2 i} O_{i} P_{2 i}+\lambda_{2}^{-2} P_{2 i} O_{d i} P_{2 i}+\lambda_{3}^{-2} P_{2 i} \bar{H}_{i} \bar{H}_{i}^{T} P_{2 i}
\end{aligned}\right.
$$

Based on Schur Lemma, multiply diag $\left\{Q_{i}^{-1}, I, I, I\right\}$ on both sides of (28), then we can obtain $\Gamma<-P_{2 i}$. Further, (34) is rewritten as

$$
\digamma \Phi_{3}(\bar{x}(t), i, t) \leq-\bar{x}^{T}(t) P_{2 i} \bar{x}(t)+\lambda_{1}^{2} \rho^{2}+\lambda_{2}^{2}+\lambda_{3}^{2} y_{h}^{2}
$$

It is easy to find that $\digamma \Phi_{3}<0$ holds, if $\bar{x}^{T}(t) P_{2 i} \bar{x}(t)>\lambda_{1}^{2} \rho^{2}+\lambda_{2}^{2}+\lambda_{3}^{2} y_{h}^{2}$ can be satisfied. Thus, for any $\bar{x}(t)$, it is easy to get that the following inequality

$$
\bar{x}^{T}(t) P_{2 i} \bar{x}(t) \leq \max \left\{\lambda_{1}^{2} \rho^{2}+\lambda_{2}^{2}+\lambda_{3}^{2} y_{h}^{2}, \bar{x}^{T}(0) P_{2 i} \bar{x}(0)\right\}
$$

It also means that the closed-loop system (5) is stochastically stable, and the state trajectory of the system can converge into the region $\Omega_{\bar{x}(t)}$.

In the following, we will discuss the dynamic tracking of the output $y(t)$ and the disturbance attenuation of $d(t)$. In particular, the $L_{1}$ performance index $\sup _{\|d(t)\|_{\infty} \leq 1}\left\|y_{d}(t)\right\|_{\infty} \leq \beta^{2}$ is imported to analyze the inhibition effect.

Theorem 4. For given parameters $\beta>0, \lambda_{1}>, \lambda_{2}>0$ and $\lambda_{3}>0$, if there exists matrices $Q_{i}=P_{2 i}^{-1}$ and $T_{2 i}, i=1,2, \cdots, N$ to make (30) and the following inequalities

$$
\left[\begin{array}{cc}
Q_{i} & Q_{i} \bar{D}_{i}^{T} \\
* & \zeta^{-1} y_{h}^{2} I
\end{array}\right]>0, \quad i=1,2, \cdots, N
$$


and

$$
\left[\begin{array}{ccc}
Q_{i} & 0 & Q_{i} \bar{D}_{i}^{T} \\
* & (\beta-\zeta) I & \bar{D}_{i}^{T} \\
* & * & \beta I
\end{array}\right]>0, \quad i=1,2, \cdots, N
$$

are solvable, then the closed-loop MJS can be proved to be stable. Meanwhile, the system output can effectively track to $y_{d}$, which is $\lim _{t \rightarrow \infty} y(t)=y_{h}$. The measurable output $y_{d}(t)$ can meet the disturbance attenuation index, which is $\sup _{\|d(t)\|_{\infty} \leq 1}\left\|y_{d}(t)\right\|_{\infty} \leq \beta^{2}$. The ISM controller gain $K_{i}$ can be solved by $K_{i}=T_{2 i} Q_{i}^{-1}$.

Proof of Theorem 4. It can be known, from Theorem 3, that the augmented closed-loop MJS is stable. Next, the problem of dynamic tracking and disturbance attenuation will be analyzed. By squaring the system output $y(t)$, one has

$$
|y(t)|^{2}=\bar{x}^{T}(t) \bar{D}_{i}^{T} \bar{D}_{i} \bar{x}(t)
$$

Based on Schur Lemma with (37), one has

$$
P_{2 i}-\zeta y_{h}^{-2} \bar{D}_{i}^{T} \bar{D}_{i} \geq 0
$$

Further, it can be infered that

$$
|y(t)|^{2} \leq \zeta^{-1} y_{h}^{2} \bar{x}^{T}(t) P_{2 i} \bar{x}(t) \leq y_{h}^{2}
$$

It is distinct that $|y(t)| \leq y_{h}$ can hold. Besides, noticing that the term $\int_{0}^{t} e(\vartheta) d \vartheta$ is one of components in $\bar{x}(t)$, we may conclude that, when $t \rightarrow \infty, \int_{0}^{t} e(\vartheta) d \vartheta$ must converge into the set $\Omega_{\bar{x}(t)}$. Therefore, we can deduce that $\lim _{t \rightarrow \infty} y(t)=y_{h}$. As a result, the good dynamical tracking can be proven.

Similarly, based on Schur complement theorem to (38), we can get

$$
\left[\begin{array}{cc}
P_{2 i} & 0 \\
0 & (\beta-\zeta) I
\end{array}\right]-\frac{1}{\beta}\left[\begin{array}{c}
\bar{D}_{d i}^{T} \\
\bar{E}_{d i}^{T}
\end{array}\right]\left[\begin{array}{cc}
\bar{D}_{d i} & \bar{E}_{d i}
\end{array}\right]>0
$$

Further, can get

$$
\beta^{-1}\left\|y_{d}(t)\right\|^{2} \leq \bar{x}^{T} P_{2 i} \bar{x}+(\beta-\zeta) \leq \beta
$$

Therefore, we can deduce that the $L_{1}$ index is certainly less than $\beta$, which equals the inequality $\sup _{\|d(t)\|_{\infty} \leq 1}\left\|y_{d}(t)\right\|_{\infty} \leq \beta^{2}$.

Remark 2. By using the above four theorems, the anti-disturbance tracking control requirement can be gradually proved. The Theorem 1 guarantees the stability of the disturbance estimation error system, which also embodies the effectiveness of the designed DO (6). By using the estimation information of disturbances, the controller and ISS are designed. Based on this, the Theorem 2 further verifies the reachability time to ISS of system state. Based on the proofs of Theorems 1 and 2, Theorem 3 gives the feasible stability proof of augmented systems. The Theorem 4 can be regarded as the final refinement of Theorem 3. The dynamical tracking and disturbance suppression problem can be successfully resolved based on Theorem 4. From Theorems 1-4, it can be seen that the multi-objective control requirement can be implemented step-by-step, which has an independent significance in the domain of control of MJSs and anti-disturbance control.

Remark 3. Please noted that this manuscript mainly focuses on the anti-disturbance algorithm design and theoretical proof. Although this manuscript includes many mathematical contents, greater attentions are the specific performance analysis and algorithm implementation. In fact, the implementation of the controller (17) and observer (6) is relatively simple. The controller gain and the observer gain can be easily computed by solving the inequalities (8) and (30). Further, 
both (8) and (30) are typical matrix inequalities, and they are not hard to compute by using the LMI toolbox. To sum up, the algorithm is easy to implement and it is fully capable of realizing some potential applications.

\section{An Simulation Example}

For verifying the effectiveness of the proposed algorithm, we consider the following MJSs with two modes suffering by unknown disturbances. The corresponding parameters are selected as

Mode 1:

$$
\begin{gathered}
A_{1}=\left[\begin{array}{cccc}
-0.0605 & 32.37 & 0 & 1 \\
-0.00014 & -1.475 & 1 & 0 \\
-0.0111 & -34.72 & -2.793 & 0 \\
0 & 0 & 1 & 0
\end{array}\right], B_{1}=\left[\begin{array}{c}
0 \\
-0.1064 \\
-33.8 \\
0
\end{array}\right], C_{1}=\left[\begin{array}{c}
0.1 \\
0 \\
-3 \\
0.1
\end{array}\right] \\
D_{1}=\left[\begin{array}{l}
1 \\
1 \\
1 \\
1
\end{array}\right], W_{1}=\left[\begin{array}{cc}
0 & 6 \\
-6 & -0.3
\end{array}\right], V_{1}=\left[\begin{array}{ll}
3 & 0
\end{array}\right]
\end{gathered}
$$

Mode 2:

$$
\begin{gathered}
A_{2}=\left[\begin{array}{cccc}
-0.0088 & -0.21 & 0 & -0.409 \\
-0.0915 & -0.5917 & 1.2 & 0 \\
-0.0294 & -2.5464 & 0.897 & 0 \\
0 & 0 & 1 & 0
\end{array}\right], B_{2}=\left[\begin{array}{c}
0.1 \\
-0.1011 \\
-7.7037 \\
0.1
\end{array}\right], C_{2}=\left[\begin{array}{c}
1 \\
0.1 \\
1.3 \\
0.1
\end{array}\right] \\
D_{2}=\left[\begin{array}{c}
1 \\
0.1 \\
1.3 \\
2
\end{array}\right], W_{2}=\left[\begin{array}{cc}
0 & 5 \\
-5 & -0.1
\end{array}\right], V_{2}=\left[\begin{array}{ll}
0.1 & 0
\end{array}\right]
\end{gathered}
$$

Let the transition rate be given as $\pi_{11}=-2, \pi_{12}=2, \pi_{21}=0.9, \pi_{22}=-0.9$. Define the parameters $\lambda_{1}=\lambda_{2}=\lambda_{3}=0.8$. Subsequently, by solving the inequalities (8), (30), and (31), the DO gains and ISM controller gains can be computed by

$$
\begin{gathered}
L_{1}=\left[\begin{array}{ccccc}
0 & -0.0697 & 0.0027 & 0.0771 & 0.0003 \\
0 & 0.0014 & -0.0001 & -0.0015 & 0
\end{array}\right] \\
L_{2}=\left[\begin{array}{ccccc}
0 & -0.0663 & 0.0028 & 0.0741 & 0 \\
0 & 0.0033 & -0.0001 & -0.0037 & 0
\end{array}\right] \\
K_{1}=\left[\begin{array}{lllll}
-1.2344 & -5.7755 & -1.3298 & -4.1037 & -0.7188
\end{array}\right] \\
K_{2}=\left[\begin{array}{lllll}
-0.9829 & -4.9555 & -1.2187 & -1.1060 & -0.5233
\end{array}\right]
\end{gathered}
$$

Assuming the primary terms as $x(0)=[20,-20,40,-20]$ and $\sigma_{1}(0)=[0.04,0.98]$. The desired output is defined as $y_{h}=30$. The switching signal is displayed in Figure 1. Figure 2 is the trajectories of system state. It can be seen that, due to the influence of initial state, the huge transients of $x_{1}$ and $x_{3}$ may occur at the beginning of the control. From the Figure 2, despite there existing the huge transient, the state of MJS can be stable very quickly by using the designed control algorithm. Figure 3 shows the dynamics of $h(t)$ and its estimation value. Figures 4 and 5, respectively, give the responses of controller input and the ISS. Figure 6 shows the dynamics of system output. The favorable disturbance estimation, output constraint, and dynamical tracking can be embodied from Figures 1-6. 


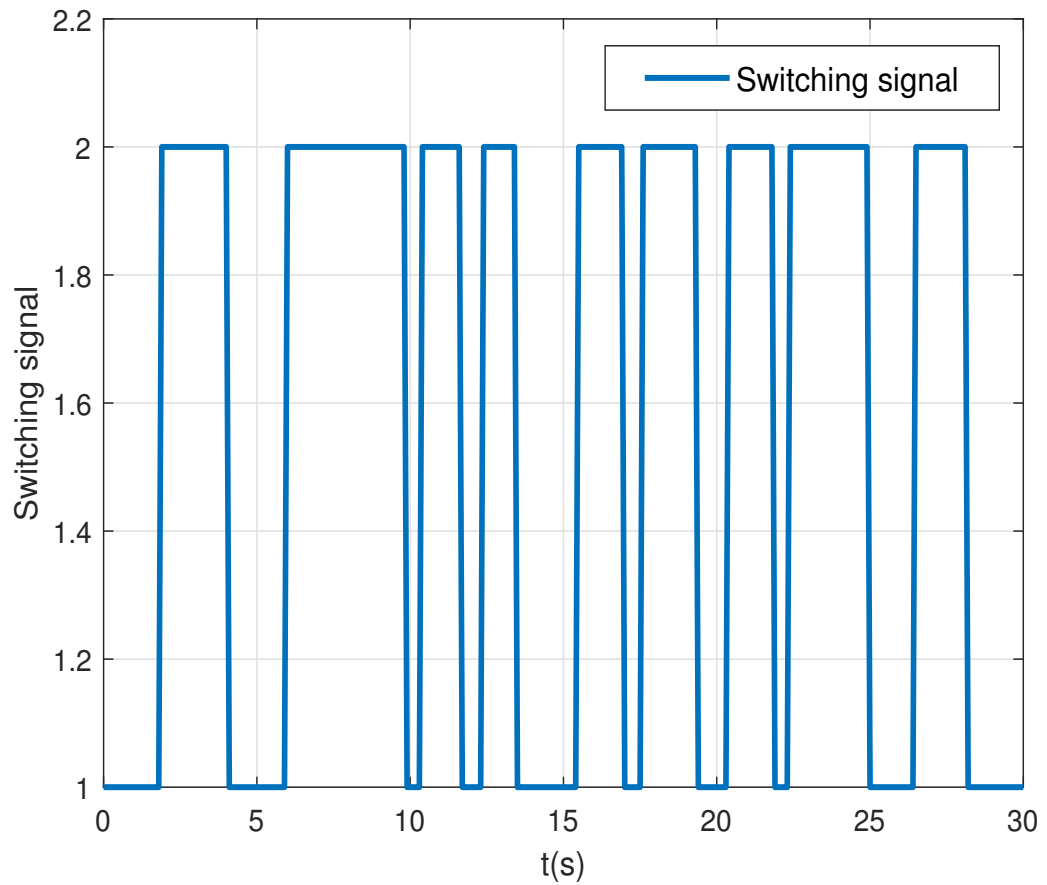

Figure 1. Switching signal.

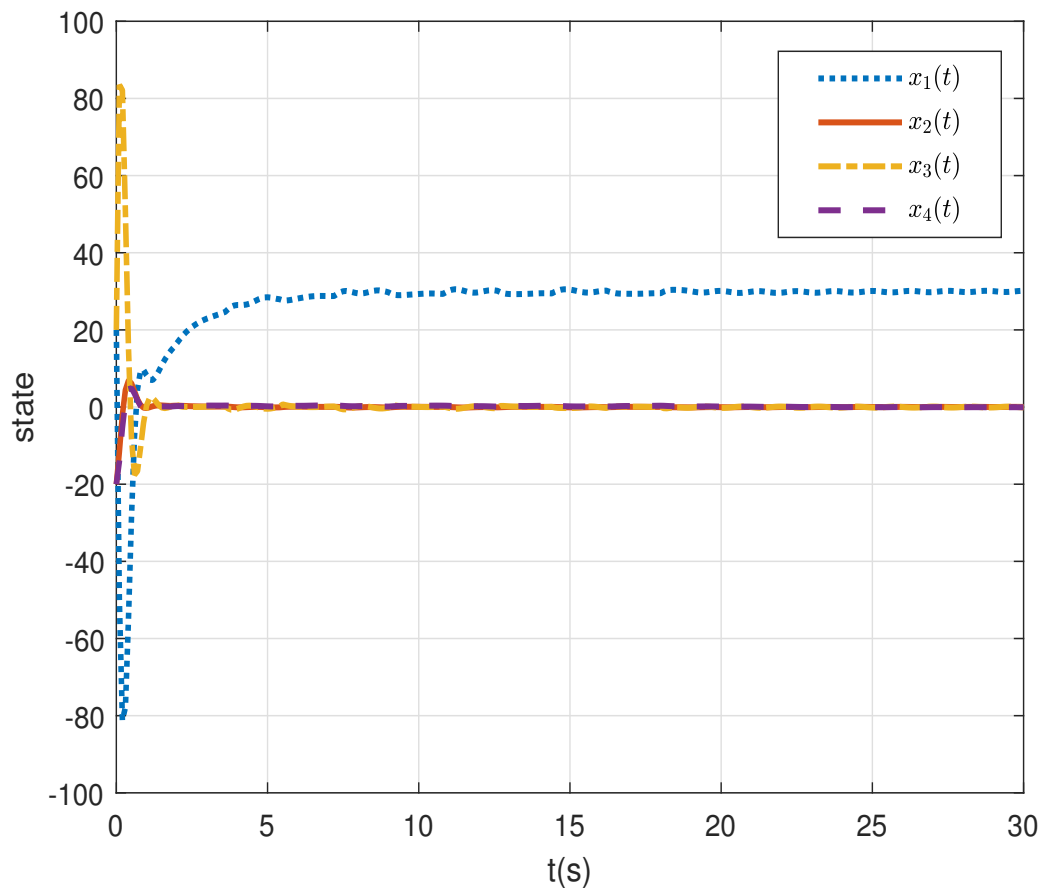

Figure 2. Trajectory of the system states. 


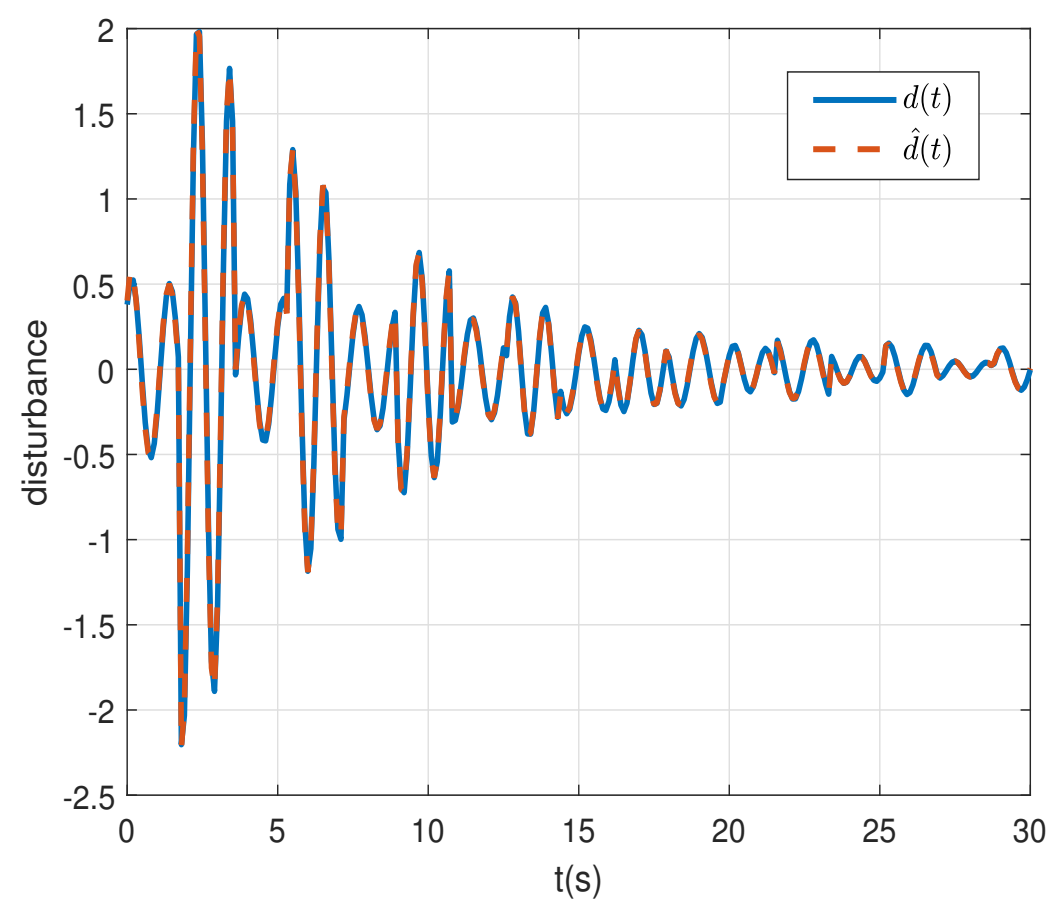

Figure 3. Disturbance and its estimation.

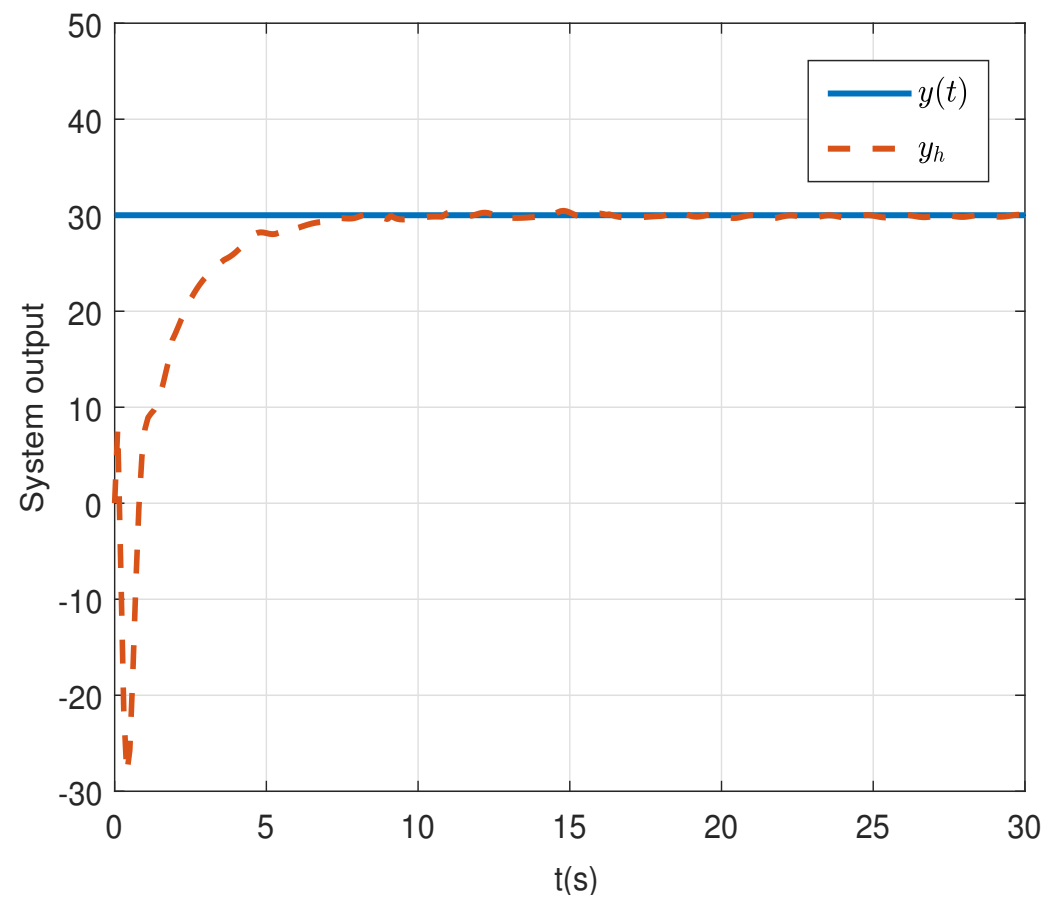

Figure 4. Trajectory of system output. 


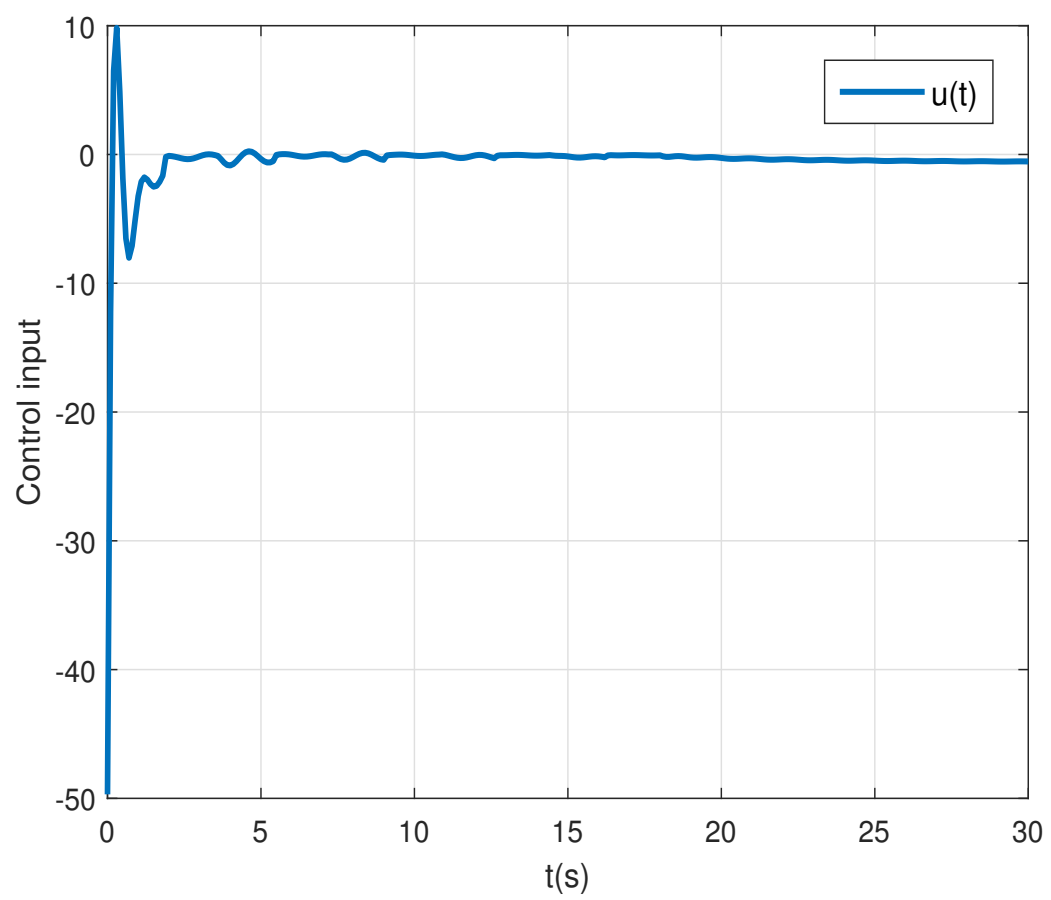

Figure 5. Dynamics of control input.

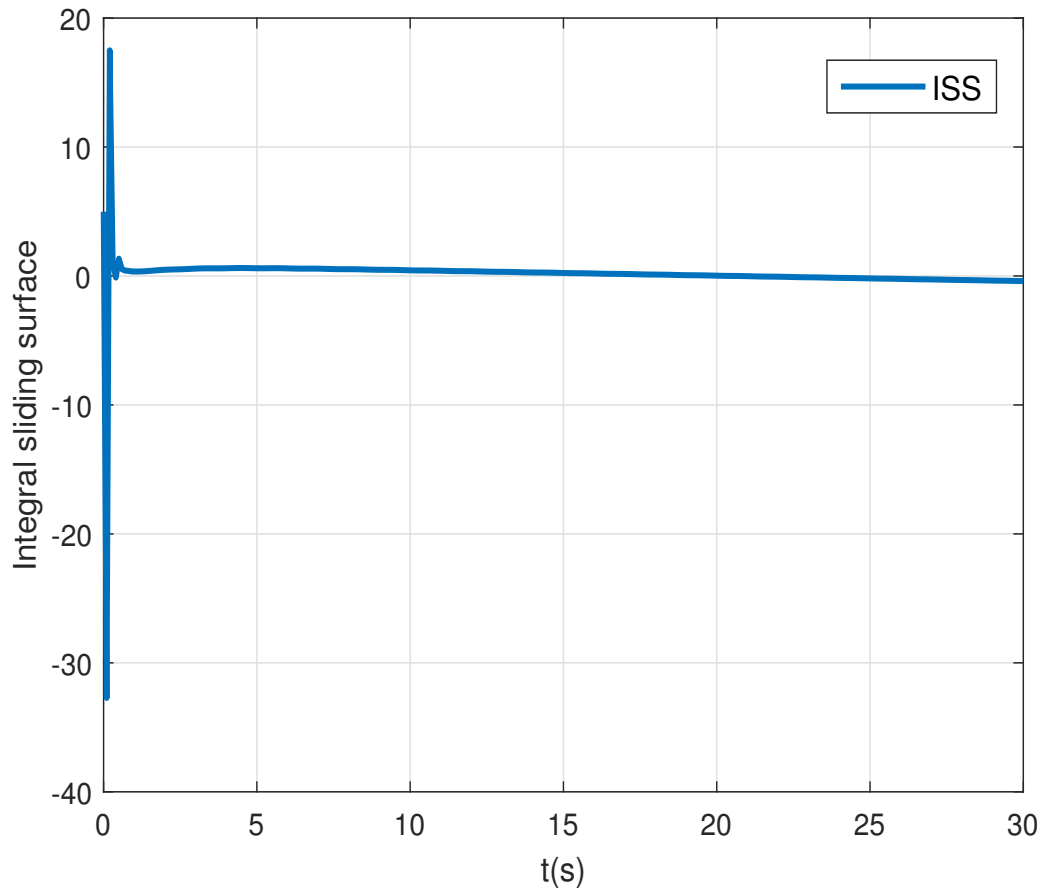

Figure 6. Integral sliding surface.

\section{Conclusions}

In this paper, an effective anti-disturbance ISM control algorithm is put forward for a typical of MJSs with mismatched disturbances. The mismatched disturbances can be dynamically estimated by constructing a nonlinear DO. Based on this, the ISS and ISM controller are both created to realize the dynamic compensation for unknown disturbances. Further, the convex optimization algorithm is designed to make the system stochastically stable. Meanwhile, the system output constraint can be guaranteed, and the tracking error ultimately converges to zero. Finally, the simulation results can verify the feasibility of the algorithm. 
Author Contributions: Conceptualization, H.S. and Y.Y.; Methodology, H.S. and Y.Y.; Software, X.Z.; Writing—original draft, X.Z.; Writing—review and editing, H.S. All authors have read and agreed to the published version of the manuscript.

Funding: This work is partially supported by the National Natural Science Foundation of China (Grant No. 61973266 and 61803331).

Conflicts of Interest: The authors declare no conflict of interest.

\section{References}

1. Costa, O.L.V.; Fragoso, M.D.; Todorow, M.G. Continuous-Time Markov Jump Linear System; Springer: London, UK, 2013.

2. Kawka, P.A.; Alleyne,A.G. Robust wireless servo control using a discrete-time uncertain Markovian jump linear model. IEEE Trans. Autom. Control 2019, 17, 733-742. [CrossRef]

3. Zhu, J.; Wang, L.P.; Spiryagin, M. Control and decision strategy for a class of Markovian jump systems in failure prone manufacturing process. IET Control Theory Appl. 2012, 6, 1803-1811. [CrossRef]

4. Al-Azzawi, W.; Al-Akaidi, M. Robust stability of solar-power wireless network control system with stochastic time delays based on $H_{\infty}$ norm. Int. J. Syst. Sci. 2015, 46, 896-907. [CrossRef]

5. Ding,D.R; Wang, Z.D.; Han, Q.L. Neural-network-based output-feedback control with stochastic communication protocols. Automatica 2019, 106, 221-229. [CrossRef]

6. Wu, L.G.; Shi,P.; Gao, H.J. State estimation and sliding-mode control of Markovian jump singular systems. IEEE Trans. Autom. Control 2010, 55, 1213-1219.

7. Nogueira, S.L.; Siqueira, A.A.G; Inoue, R.S; Terra, M.H. Markov jump linear systems-based position estimation for lower limb exoskeletons. Sensors 2014, 14, 1835-1849. [CrossRef]

8. Shi, P.; Liu, M.; Zhang, L.X. Fault-tolerant sliding-mode-observer synthesis of Markovian jump systems using quantized measurements. IEEE Trans. Ind. Electron. 2015, 62, 5910-5918. [CrossRef]

9. Qi, W.H.; Zong, G.D.; Karimi, H.R. Sliding mode control for nonlinear stochastic singular semi-Markov jump systems. IEEE Trans. Autom. Control 2020, 65, 361-368. [CrossRef]

10. Wu, Z.G.; Dong, S.L.; Shi, P.; Su, H.Y.; Huang, T.W. Reliable filtering of nonlinear Markovian jump systems: The continuous-time case. IEEE Trans. Syst. Man Cybern. Syst. 2019, 49, 386-394. [CrossRef]

11. Aberkane, S. Bounded real lemma for nonhomogeneous Markovian jump linear systems. IEEE Trans. Autom. Control 2013, 58, 797-801. [CrossRef]

12. Shen,H.;Men, Y.Z.; Wu, Z.G.; Park, J.H. Nonfragile $H_{\infty}$ control for fuzzy markovian jump systems under fast sampling singular perturbation. IEEE Trans. Fuzzy Syst. 2018, 48, 2058-2069.

13. Shen,M.Q.; Ma,Y.S.; Park,J.H; Wang, Q.G. Fuzzy tracking control for Markov jump systems with mismatched faults by iterative proportional-integral observers. IEEE Trans. Fuzzy Syst. 2020. [CrossRef]

14. Wang,Y.Y.; Xia,Y.Q.; Shen,H.; Zhou,P.F. SMC design for robust stabilization of nonlinear Markovian jump singular systems. IEEE Trans. Autom. Control 2018, 63, 219-224. [CrossRef]

15. Guo. L; Cao, S.Y. Anti-Disturbance Control for Systems with Multiple Disturbances; CRC Press: Boca Raton, FL, USA, 2013.

16. Zhang, Y.L.; Zhu,M.; Li, D.; Wang, J.M. ADRC dynamic stabilizabtion of an unstable heat equation. IEEE Trans. Autom. Control 2020, 65, 4424-4429. [CrossRef]

17. Xia, Y.Q.; Pu, F.; Li, S.F.; Gao, Y. Lateral path tracking control of autonomous land vehicle based on ADRC and differential flatness. IEEE Trans. Ind. Electron. 2016, 63, 3091-3099. [CrossRef]

18. Yu, P.; Liu, K.Z.; Wu, M.; She, J. Improved equivalent-inputbdisturbance approach based on $H_{\infty}$ control. IEEE Trans. Ind. Electron. 2020, 67, 8670-8679. [CrossRef]

19. Chen, W.H. Disturbance observer based control for nonlinear systems. IEEE/ASME Trans. Mech. 2020, 9, 706-710. [CrossRef]

20. Sun, H.B.; Guo, L. Neural network-based DOBC for a class of nonlinear systems with unmatched disturbances. IEEE Trans. Neural Netw. Learn. Syst. 2017, 28, 482-489. [CrossRef]

21. Chen,W.H.; Ballanceand, D.J.; Gawthrop, P.J. A nonlinear disturbance observer for robotic manipulators. IEEE Trans. Ind. Elect. 2000, 47, 932-938. [CrossRef]

22. Sun, L.; Huo, W.; Jiao, Z.X. Disturbance-observer-based robust relative pose control for spacecraft rendezvous and proximity operations under input saturation. IEEE Trans. Aerosp. Electron. Syst. 2018, 54, 1605-1617. [CrossRef]

23. Liu, Y.; Fu, Y.; He, W.; Hui, Q. Modeling and observer-based vibration control of a flexible spacecraft with external disturbances. IEEE Trans. Ind. Electron. 2019, 66, 8648-8658. [CrossRef]

24. Zhang, X.G.; Li, Z.X. Sliding-mode observer-based mechanical parameter estimation for permanent magnet synchronous motor. IEEE Trans. Power Electron. 2016, 31, 5732-5745. [CrossRef]

25. Yang, J.; Cui, H.Y.; Li, S.H.; Zolotas, A. Optimized active disturbance rejection control for DC-DC buck converters with uncertainties using a reduced-order GPI observer. IEEE Trans. Circuits Syst. I Reg. Pap. 2018, 65, 832-841. [CrossRef]

26. Yi, Y.; Zheng, W.X.; Sun, C.Y.; Guo, L. DOB fuzzy controller design for non-Gaussian stochastic distribution systems using two-step fuzzy identification. IEEE Trans. Fuzzy Syst. 2016, 24, 401-418. [CrossRef] 
27. Yi, Y.; Zheng, W.X.; Liu, B. Adaptive anti-disturbance control for systems with saturating input via dynamic neural network disturbance modeling. IEEE Trans. Cybern. 2020. [CrossRef]

28. Kim,K.S.; Rew, K.H.; Kim, S. Disturbance observer for estimating higher order disturbances in time series expansion. IEEE Trans. Autom. Control 2010, 55, 1905-1911.

29. Yao, X.M.; Park, J.H.; Wu, L.G.; Guo, L. Disturbance-observer-based composite hierarchical anti-disturbance control for singular Markovian jump systems. IEEE Trans. Autom. Control 2019, 64, 2875-2882. [CrossRef]

30. Freidovich, L.B.; Khalil, H.K. Performance recovery of feedback-linearization-based designs. IEEE Trans. Autom. Control 2008, 53, 2324-2334. [CrossRef]

31. Chowdhury, D.; Khalil, H.K. Practical synchronization in networks of nonlinear heterogeneous agents with application to power systems. IEEE Trans. Autom. Control 2020, 66, 184-198. [CrossRef]

32. Utkin, V. Variable structure systems with sliding modes. IEEE Trans. Autom. Control 1977, 22, 212-222. [CrossRef]

33. Edwards, C.; Spurgeon, S. Sliding Mode Control: Theory and Application; Taylor and Francis: London, UK, 1998.

34. Ding, S.H.; Mei, K.Q.; Li, S.H. A new second-order sliding mode and its application to nonlinear constrained systems. IEEE Trans. Autom. Control 2019, 64, 2545-2552. [CrossRef]

35. Zhang, Q.; Zhao, X.; Liu, L.; Dai, T. Adaptive sliding mode neural network control and flexible vibration suppression of a flexible spatial parallel robot. Electronics 2021, 10, 212. [CrossRef]

36. Huynh, V.V.; Minh, B.L.N.; Amaefule, E.N.; Tran, A.T.; Tran, P.T. Highly robust observer sliding mode based frequency control for multi area power systems with renewable power plants. Electronics 2021, 10, 274. [CrossRef]

37. Xu, S.S.; Chen, C.; Wu, Z. Study of nonsingular fast terminal sliding-mode fault-tolerant control. IEEE Trans. Ind. Electron. 2015, 62, 3906-3913. [CrossRef]

38. Wang, J.H.; Xu, Y.L.; Xu. Y.; Yang, D.D. Time-varying formation for high-order multi-agent systems with external disturbances by event-triggered integral sliding mode control. Appl. Math. Comput. 2019, 359, 333-343. [CrossRef]

39. Wang, Y.Y.; Shen, H.; Karimi, H.R.; Duan, D. Dissipativity-based fuzzy integral sliding mode control of continuous-time T-S fuzzy systems. IEEE Trans. Fuzzy Syst. 2018, 26, 1164-1176. [CrossRef]

40. Lee, J.; Chang, P.H.; Jin, M. Adaptive integral sliding mode control with time-delay estimation for robot manipulators. IEEE Trans. Ind. Electron. 2017, 64, 6796-6804. [CrossRef]

41. Yang, C.Y.; Che, Z.Y.; Fu, J.; Zhou, L.N. Passivity-based integral sliding mode control and $\varepsilon$-bound estimation for uncertain singularly perturbed systems with disturbances. IEEE Trans. Circuits Syst. II Exp. Briefs 2019, 66, 3906-3913. 